\title{
A TWO-DIMENSIONAL MIXED BOUNDARY VALUE PROBLEM IN ELASTICITY*
}

By A. M. ROBERTS (Monash University, Victoria, Australia)

1. Introduction. The problem of the two-dimensional indentation of an elastic halfspace $(y \leq 0)$ by a rigid stamp of given profile (Hertz problem) has been discussed in the standard texts [1], [2], and [3]. The more involved problem of indentation with friction (which includes the Hertz problem as a special case), given by

$$
\begin{gathered}
\tau_{\nu \nu}(x, 0)=0, \quad \tau_{x y}(x, 0)=0, \quad x<-b, \quad x>a, \\
v(x, 0)=g(x), \quad \tau_{x y}(x, 0)=\lambda \tau_{\nu y}(x, 0), \quad-b<x<a,
\end{gathered}
$$

where $\tau$ denotes stress, $v$ is the displacement in the $y$ direction and $\lambda$ the coefficient of friction, has also been fully discussed in these books. The present note gives a solution to the boundary value problem

$$
\begin{gathered}
\tau_{y y}(x, 0)=0, \quad \tau_{x y}(x, 0)=0, \quad x<-b, \quad x>a, \\
v(x, 0)=g(x), \quad \tau_{x y}(x, 0)=\lambda \tau_{y y}(x, 0), \quad-b<x<0, \\
v(x, 0)=g(x), \quad \tau_{x y}(x, 0)=-\lambda \tau_{y y}(x, 0), \quad 0<x<a,
\end{gathered}
$$

which is a generalization of (1) relevant to a stamp of curved or pointed profile loaded vertically, where friction acts in opposing directions on opposite sides of the stamp. In accordance with the classical Hertz problem, we regard the resultant force $(X,-Y)$ exerted by the stamp as known, and the width of indentation given by $a$ and $b$ as unknown and to be determined in terms of this force.

2. General solution. The changeover in conditions (2) at $x=0$ means that the construction of solution functions as Cauchy integrals will in general lead to integrals which cannot be explicitly evaluated. Certain particular surface profiles $g(x)$, however, lead to closed-form solutions. We first let

$$
\tau_{y y}(x, 0)=f_{1}(x), \quad \tau_{x y}(x, 0)=f_{2}(x), \quad-b<x<a,
$$

and define the (unknown) functions $w_{1}$ and $w_{2}$ of the complex variable $z=x+i y$ by

$$
w_{1,2}(z)=\int_{-b}^{a} f_{1,2}(t) /(t-z) \cdot d t
$$

It follows from the Plemelj formulae for functions defined as Cauchy integrals that

$$
\begin{aligned}
w_{1,2}^{\mp}(x) & =w_{1,2}(x \mp i 0) \\
& =\mp \pi i f_{1,2}(x)+\int_{-b}^{a} f_{1,2}(t) /(t-x) \cdot d t,
\end{aligned}
$$

where the integral is interpreted as a Cauchy principal value. Conditions (5) may now be used in the relations (2) to obtain a Riemann boundary value problem for $w_{\mathbf{1}}$.

* Received July 10, 1969. 
The relation [1, Eq. 93.6],

$$
-2 \pi i \Phi(z)=w_{1}(z)-i w_{2}(z)
$$

links the functions $w$ with the potential $\Phi$ of Muskhelishvili's work. Taking the complex conjugate of (6) (denoted by a bar) and replacing $\bar{z}$ by $z$ gives

$$
2 \pi i \Phi(z)=\bar{w}_{1}(z)+i \bar{w}_{2}(z) .
$$

Eqs. (6) and (7) combine to be equivalent to the extension across the real axis used by Muskhelishvili to express his boundary value problems in terms of the potential $\Phi$ alone. We also have $[1,112.15]$

$$
2 \mu^{\prime}(\partial / \partial x)(u+i v)=\kappa \Phi(z)+\Phi(\bar{z})-(z-\bar{z}) \overline{\Phi^{\prime}(z)},
$$

where $\kappa$ is related to the Lamé constants $\lambda^{\prime}$ and $\mu^{\prime}$ by

$$
\kappa=\left(\lambda^{\prime}+3 \mu^{\prime}\right) /\left(\lambda^{\prime}+\mu^{\prime}\right),
$$

and the prime on $\Phi$ indicates differentiation. Using Eqs. (6) and (5) in (8) leads to

$$
\begin{aligned}
\left.2 \mu^{\prime}(\partial / \partial x)(u+i v)\right|_{y=0-}= & \frac{1}{2}(\kappa-1) \cdot\left[f_{1}(x)-i f_{2}(x)\right] \\
& -(\kappa+1) /(2 \pi i) \cdot \int_{-b}^{a}\left[f_{1}(t)-i f_{2}(t)\right] /(t-x) \cdot d t .
\end{aligned}
$$

Extraction of the imaginary part of $(9)$ and substitution of $v(x, 0)$ from (2) gives $2 \mu^{\prime} g^{\prime}(x)=(\kappa+1) /(2 \pi) \cdot \operatorname{Re} w_{1}^{-}(x)+(\kappa-1) /(2 \pi) \cdot \operatorname{Im} w_{2}^{-}(x), \quad-b<x<a$.

The stress boundary conditions in $-b<x .<a$ lead to

$$
f_{2}(x)= \pm \lambda f_{1}(x), \quad-b<x<0 \text { and } 0<x<a, \quad \text { respectively. }
$$

This condition cnables us to eliminate $\operatorname{Im} w_{2}^{-}$from (10), giving the following Riemann problem in $w_{1}$ :

$$
\begin{gathered}
(\kappa+1) \cdot \operatorname{Re} w_{1}^{-}(x)+\lambda(\kappa-1) \cdot \operatorname{Im} w_{1}^{-}(x)=4 \pi \mu^{\prime} g^{\prime}(x), \quad-b<x<0, \\
(\kappa+1) \cdot \operatorname{Re} w_{1}^{-}(x)-\lambda(\kappa-1) \cdot \operatorname{Im} w_{1}^{-}(x)=4 \pi \mu^{\prime} g^{\prime}(x), \quad 0<x<a, \\
\operatorname{Im} w_{1}^{-}(x)=0, \quad x<-b, \quad x>a .
\end{gathered}
$$

Our method of solving (12) for $w_{1}(z)$ is basically that of Galin [3, §4]. Defining $\alpha$ and $Q$ by

$$
\begin{gathered}
\tan \pi \alpha=\lambda(\kappa-1) /(\kappa+1), \quad 0 \leq \alpha<\pi / 2, \\
Q=\left[(\kappa+1)^{2}+\lambda^{2}(\kappa-1)^{2}\right]^{1 / 2},
\end{gathered}
$$

we may rewrite (12) as

$$
\begin{array}{rlrl}
\operatorname{Im}\left[i \cdot e^{-i \pi \alpha} \cdot w_{1}^{-}(x)\right] & =4 \pi \mu^{\prime} g^{\prime}(x) / Q, & -b<x<0 . \\
\operatorname{Im}\left[i \cdot e^{i \pi \alpha} \cdot w_{1}^{-}(x)\right] & =4 \pi \mu^{\prime} g^{\prime}(x) / Q, & 0<x<a, \\
\operatorname{Im} w_{1}^{-}(x) & =0, \quad x<-b, \quad x>a .
\end{array}
$$

The solution to the homogeneous problem corresponding to (15) is

$$
w_{1}^{0}(z)=(z-a)^{1 / 2+\alpha} \cdot(z+b)^{1 / 2+\alpha} \cdot z^{-2 \alpha-2},
$$


as may be easily verified by substitution into (15) with the right sides all zero. The branch cuts of the surds are from $-b$ to 0 and from 0 to $a$ along the real $z$ axis, and the branch chosen is that which behaves like $1 / z$ for large $|z|$. Expression (16) is also chosen to vanish at $-b$ and $a$ (an infinity at either of these points leads to an infinite stress there, which occurs only if the punch has a corner), and to vanish at infinity. The singularity at the origin will be dealt with later.

If we divide (15) through by the real quantity $\left|w_{1}^{0-}(x)\right|$, we may absorb the exponential factors on the left sides to obtain

$$
\begin{aligned}
\operatorname{Im}\left[w_{1}^{-}(x) / w_{1}^{0-}(x)\right] & =4 \pi \mu^{\prime} g^{\prime}(x) /\left(Q\left|w_{1}^{0-}(x)\right|\right), \quad-b<x<a \\
& =0 \quad x<-b, \quad x>a
\end{aligned}
$$

which has immediate solution

$$
\begin{aligned}
w_{1}(z)= & -4 \mu^{\prime} / Q \cdot w_{1}^{0}(z) \cdot \int_{-b}^{a} g^{\prime}(t) /(t-z) \cdot d t /\left|w_{1}^{0-}(t)\right|+c_{0} w_{1}^{0}(z) \\
= & (z-a)^{1 / 2+\alpha} \cdot(z+b)^{1 / 2+\alpha} \cdot z^{-2 \alpha-2} \\
& \cdot\left\{Y-4 \mu^{\prime} / Q \cdot \int_{-b}^{a} g^{\prime}(t) /(t-z) \cdot|t|^{2 \alpha+2} \cdot|t-a|^{-1 / 2-\alpha} \cdot|t+b|^{-1 / 2-\alpha} \cdot d t\right\} .
\end{aligned}
$$

The constant $c_{0}$ has been taken equal to the resultant downward force $Y$, since it is the coefficient of $1 / z$ for large $|z|$ in (18), which may be compared with the expansion of (4) for large $|z|$. Expression (18) is the general solution to our problem. It is not possible to obtain an expression for $w_{2}(z)$ directly, as Re $w_{1}^{-}$cannot be eliminated from (10). Having solved for $w_{1}$, the surface stress $f_{1}$ may be found, so that $f_{2}$ follows by (11), and a formal expression for $w_{2}$ follows from (4). The density function $f_{2}$ in this integral will be discontinuous at $t=0$, by (11).

3. Particular solution. If $g^{\prime}(t)$ is a polynomial, expression (18) may be explicitly evaluated. For the case of a $V$-shaped stamp, $g^{\prime}$ is discontinuous, and simplification is only possible for symmetric indentation. Application of the method used by Muskhelishvili to solve problem (1) leads to an expression similar to (15) for the function $\Phi$ in the present problem, but our $g^{\prime}(x)$ is replaced by

$$
(1 \pm i \lambda) \cdot g^{r}(x), \quad-b<x<0, \quad 0<x<a,
$$

which is not as easily simplified as our expressions.

The most simple curved stamp profile is circular, which may be considered as an approximation to any smoothly curved stamp with radius of curvature $R$. In this case,

$$
g(x)=x^{2} /(2 R)-d
$$

and we may evaluate (18) by contour integration. We have

$$
\oint_{C} F(\zeta) /(\zeta-z) \cdot d \zeta=2 \pi i \cdot F(z)
$$

where

$$
F(\zeta)=\zeta^{2 \alpha+3} \cdot(\zeta+b)^{-1 / 2-\alpha} \cdot(\zeta-a)^{-1 / 2-\alpha},
$$

and $C$ is a circle of large radius with an indentation along the real $\zeta$ axis going round the branch cut linking $-b$ and $a$. The left-hand side of (20) contributes a residue at infinity, 
plus two branch integrals of the form of (18), with $g$ given by (19). We obtain

$$
\begin{aligned}
w_{1}(z)= & 4 \pi \mu^{\prime} z /[R(\kappa+1)]\left\{1-z^{-2 \alpha-3}(z-a)^{1 / 2+\alpha}(z+b)^{1 / 2+\alpha}\right. \\
& \cdot\left[z^{2}-z(a-b)\left(\frac{1}{2}+\alpha\right)-\frac{1}{2}\left(\frac{1}{2}+\alpha\right)\left(a^{2}+b^{2}\right)\right. \\
& \left.\left.-\frac{1}{2}\left(\frac{1}{2}+\alpha\right)^{2}(a-b)^{2}+Y R(\kappa+1) /\left(4 \pi \mu^{\prime}\right)\right]\right\} .
\end{aligned}
$$

The surface stress follows from (22) by letting $y \rightarrow 0-$, noting that the branches of the surds are the same as those given for (16).

$$
\begin{aligned}
\tau_{\nu y}(x, 0)=- & 1 / \pi \cdot \operatorname{Im} w_{1}^{-}(x) \\
= & -4 \mu^{\prime} /(R Q) \cdot|x|^{-2 \alpha-2} \cdot|x-a|^{1 / 2+\alpha} \cdot|x+b|^{1 / 2+\alpha} \\
& \cdot\left\{x^{2}-x(a-b)\left(\frac{1}{2}+\alpha\right)-\frac{1}{2}\left(\frac{1}{2}+\alpha\right)\left(a^{2}+b^{2}\right)\right. \\
- & \left.\frac{1}{2}\left(\frac{1}{2}+\alpha\right)^{2}(a-b)^{2}+Y R(\kappa+1) /\left(4 \pi \mu^{\prime}\right)\right\} \quad-b<x<a .
\end{aligned}
$$

The singularity in this stress at $x=0$ must be at worst integrable, so we require

$$
(a-b)\left(\frac{1}{2}+\alpha\right)=0 \text {, }
$$

and

$$
R Y(\kappa+1) /\left(4 \pi \mu^{\prime}\right)=\frac{1}{2}\left(\frac{1}{2}+\alpha\right) \cdot\left[\left(a^{2}+b^{2}\right)+\left(\frac{1}{2}+\alpha\right)(a-b)^{2}\right] .
$$

Expression (24) requires that the indentation be symmetric, and so (25) then reduces to

$$
a^{2}=R Y /\left(4 \pi \mu^{\prime}\right) \cdot(\kappa+1) /\left(\frac{1}{2}+\alpha\right),
$$

which relates the width of indentation to the applied force. This result reduces to that of the frictionless case given in [1, Eq. 116.10a] as $\alpha \rightarrow 0$. The presence of friction slightly reduces the width of indentation through the $\frac{1}{2}+\alpha$ term. For the situation where $\lambda^{\prime}=\mu^{\prime}$, $\alpha$ only ranges from 0 to 0.108 as $\lambda$ changes from 0 to 1 . It also follows now from symmetry that the horizontal applied force $X$ must be zero. The form of (22) appropriate to the present problem may now be written as

$$
w_{1}(z)=4 \pi \mu^{\prime} / R \cdot z /(\kappa+1) \cdot\left[1-\left(1-a^{2} / z^{2}\right)^{1 / 2+\alpha}\right] .
$$

To obtain an expression for $w_{2}(z)$, we use (23) and (11) in the symmetric case to give

$$
\begin{aligned}
\tau_{x y}(x, 0)= & f_{2}(x)= \pm \lambda f_{1}(x)= \pm \lambda \tau_{y y}(x, 0) \\
= & \mp 4 \lambda \mu^{\prime} /(R Q) \cdot\left(a^{2}-x^{2}\right)^{1 / 2+\alpha} \cdot|x|^{-2 \alpha} \\
& -a<x<0, \quad 0<x<a, \quad \text { respectively. }
\end{aligned}
$$

Hence, by (4), after simplification

$$
\begin{aligned}
w_{2}(z)=-4 \lambda \mu^{\prime} /(R Q) \cdot a^{3} / z^{2} \cdot \int_{0}^{1}(1-u)^{1 / 2+\alpha} \cdot u^{-\alpha} \cdot\left(1-u a^{2} / z^{2}\right)^{-1} \cdot d u \\
=-4 \lambda \mu^{\prime} /(R Q) \cdot a^{3} / z^{2} \cdot \Gamma(1-\alpha) \cdot \Gamma(3 / 2+\alpha) / \Gamma(5 / 2) \\
\qquad F\left(1,1-\alpha ; 5 / 2 ; a^{2} / z^{2}\right) \quad|z|>a,
\end{aligned}
$$

where the hypergeometric function $F$ is defined by the preceding integral, which is of the Euler type. Using the analytic continuation of this function to give a solution near $z=0$, we find that 


$$
\begin{aligned}
w_{2}(z)= & 8 \lambda \mu^{\prime} a /(R Q) \cdot \pi^{-1 / 2} \cdot \Gamma(3 / 2+\alpha) \cdot \Gamma(-\alpha) \cdot F\left(1,-\frac{1}{2} ; 1+\alpha ; z^{2} / a^{2}\right) \\
& +4 \pi \mu^{\prime} / R \cdot\left(a^{2}-z^{2}\right)^{1 / 2} /(\kappa-1) \cdot\left(1-a^{2} / z^{2}\right)^{\alpha} \quad|z|<a,
\end{aligned}
$$

where the square root has its branch cuts on $\operatorname{Im} z=0,|\operatorname{Re} z|>a$, and is real positive when $z$ is real, while the other surd has branch cuts along the real axis in $-a<\operatorname{Re} z<0$, and $0<\operatorname{Re} z<a$, and the branch chosen is that which behaves like +1 for large $|z|$. Expressions (29) and (30) agree with the boundary conditions for $w_{2}^{-}(x)$.

A singularity such as that at the origin is usually associated with a corner of the stamp, but in the present case it results from a change in the type of boundary conditions. As $\alpha \rightarrow 0$, this singularity disappears as would be expected for $\lambda \rightarrow 0$. It follows from (9), (27) and (30) that $(\partial u / \partial x)(x, 0)$ is an even function of $x$ in $|x|<a$, with an integrable infinity of order $2 \alpha$ at the origin. The horizontal surface displacement $u(x, 0)$ will thus be bounded at the origin and be an odd function of $x$. This means that the stamp is pushing the surface of the half-space apart. It therefore appears that a change in the direction of the friction force beneath a stamp may contribute to cracking due to a buildup of stress and horizontal displacement at the center of the stamp.

The solution for a wedge shaped stamp may be obtained by putting

$$
\begin{array}{rlrl}
g(x) & =m x & 0 & <x<a, \\
& =-m x & -a & <x<0
\end{array}
$$

in formula (18). It is only possible to evaluate the resulting integral in closed form when the indentation is symmetric (and hence when the applied horizontal force is zero). We find that $w_{1}(z)$ now contains a hypergeometric function, but still has the same type of singularities as did expression (22). The requirement of an integrable singularity in stress at the origin now leads to the condition

$$
a=Y Q \pi^{1 / 2} /\left(8 \mu^{\prime} m\right) \cdot 1 / \Gamma(\alpha+1) \cdot 1 / \Gamma\left(\frac{1}{2}-\alpha\right)
$$

which is the analogue of (26). It is not possible to obtain a simple expression for $w_{2}(z)$ for this problem, but since the singularities are of the same order as those for a smoothly curved stamp, it is likely that the previous results will also hold for a pointed wedge.

\section{REFERENCES}

[1] N. I. Muskhelishvili, Some fundamental problems in the mathematical theory of elasticity. Fundamental equations, plane theory of elasticity, torsion and bending, 4th ed., Izdat. Akad. Nauk SSSR, Moscow, 1949; English transl., Noordhoff, Groningen, 1963

[2] A. E. Green and W. Zerna, Theoretical elasticity, Clarendon Press, Oxford, 1954

[3] L. A. Galin, Contact problems of the theory of elasticity, GITTL, Moscow 1953 